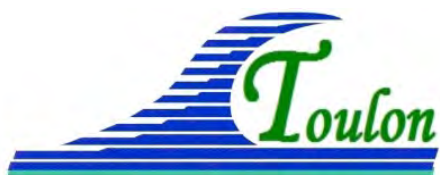

XIV èmes Journées Nationales Génie Côtier - Génie Civil Toulon, 29 juin au $1^{\text {er }}$ juillet 2016

DOI:10.5150/jngcgc.2016.056 C Cditions Paralia CFL disponible en ligne - http://www.paralia.fr - available online

\title{
Projet EMACOP : caractérisation des vagues et du potentiel houlomoteur des sites d'Esquibien et de Saint-Guénolé par simulation numérique
}

\author{
Bertrand MICHARD ${ }^{1}$, Emmanuel COSQUER ${ }^{1}$, Antoine MALLEGOL ${ }^{2}$, \\ Jonathan COIGNARD ${ }^{3}$, Jean-François FILIPOT ${ }^{4}$, \\ Komlan A. KPOGO-NUWOKLO ${ }^{5}$, Michel OLAGON ${ }^{5}$, Philippe SERGENT $^{6}$
}

1. Cerema / DTecEMF, Technopôle Brest Iroise, BP 5, 29280 Plouzané, France. bertrand.michard@cerema.fr ; emmanuel.cosquer@cerema.fr

2. Ecole Nationale des Ingénieurs de Brest, Parvis Blaise Pascal, 29280 Plouzané, France. antoine.mallegol@laposte.net

3. Université de Technologie de Compiègne, 15 rue Couttolenc, 60200 Compiègne, France.jonathan.coignard@gmail.com

4. France Energies Marines, 15 rue Johannes Kepler, 29280 Plouzané, France. jean.francois.filipot@france-energies-marines.org

5. Ifremer, Centre de Bretagne, ZI Pointe du Diable, CS 10070, 29280 Plouzané., France komlan.kpogo.nuwoklo@ifremer.fr ; michel.olagnon@ifremer.fr

6. Cerema / DTecEMF, 134 rue de Beauvais, CS 60039, 60280 Margny lès Compiègne, France.phillipe.sergent@cerema.fr

\section{Résumé :}

Un des objectifs du projet de recherche EMACOP (Energies MArines, COtières et Portuaires) est de caractériser le potentiel houlomoteur près des structures portuaires et côtières, comme les digues et jetées. L'article présente l'application d'un modèle nonhydrostatique de propagation et de transformation des vagues en proche côtier SWASH pour deux sites exposés de Bretagne. Les simulations numériques sont effectuées avec SWASH pour les conditions de houle dominante et trois niveaux de marée. Les résultats présentés ici permettent de caractériser le potentiel houlomoteur et de définir les zones énergétiques propices à l'installation de systèmes de récupération sur les deux sites.

Mots-clés : Potentiel houlomoteur, Modèle numérique, SWASH, Bretagne, EMACOP.

\begin{abstract}
:
The French research EMACOP project aims at characterising wave power nearby onshore structures. This paper presents the application of the non-hydrostatic wave-flow model SWASH to wave propagation and transformation on two hot spots in Brittany. The numerical simulations were performed for dominant wave conditions and three tide levels. The results of wave simulations allow us to characterise wave energy resources and define Wave Energy Converters (WEC)'s promising positions on both sites.
\end{abstract}

Keywords: Wave power, Resources, Numerical model, SWASH, Brittany, EMACOP. 


\section{Introduction}

Dans le cadre du projet EMACOP, un des objectifs du thème "Systèmes houlomoteurs sur ouvrages anciens" est d'étudier la ressource énergétique des vagues près des structures côtières (http://www.emacop.fr/). Une méthode de calcul analytique a permis d'identifier et de caractériser le potentiel houlomoteur de vingt-deux sites en Manche et Atlantique et de retenir les sites les plus pertinents (MICHARD et al., 2014). Pour estimer plus précisément le potentiel prometteur sur ces sites, il a été décidé d'utiliser des modèles numériques de propagation plus performants. Nous présentons ici la caractérisation des vagues et du potentiel de deux sites de Bretagne, Esquibien et SaintGuénolé, par simulation numérique des vagues à l’aide du modèle SWASH.

\section{Modèle SWASH de simulation des vagues}

\subsection{Présentation du modèle numérique non-hydrostatique}

SWASH, un acronyme de Simulating WAves till SHore, est un modèle numérique de transformation de vagues en proche côtier développé par l'Université de Technologie de Delft (NL), qui offre la possibilité d'étudier la propagation des vagues du large à la côte de façon déterministe. Le modèle résout les équations non linéaires en faible profondeur en y ajoutant un terme de pression non-hydrostatique (ZIJLEMA et al., 2011).

Le modèle SWASH prend en compte de nombreux phénomènes affectant les vagues en proche côtier, comme la propagation, la dispersion de fréquence, le levage ou shoaling, la réfraction par le fond, la diffraction, le déferlement, la friction sur le fond, les réflexions et les interactions non linéaires des vagues. Une description du modèle, du schéma numérique explicite retenu, des conditions aux limites de forçages et des applications, sont présentés sur le site dédié (http://swash.sourceforge.net).

\section{2 Équations résolues du modèle}

Les équations non linéaires en faible profondeur qui incluent un terme de pression nonhydrostatique, dérivent des équations de Navier-Stokes :

$$
\begin{aligned}
& \frac{\partial \zeta}{\partial t}+\frac{\partial h u}{\partial x}+\frac{\partial h v}{\partial y}=0 \\
& \frac{\partial u}{\partial t}+u \frac{\partial u}{\partial x}+v \frac{\partial u}{\partial y}+g \frac{\partial \zeta}{\partial x}+\frac{1}{h} \int_{-d}^{\zeta} \frac{\partial q}{\partial x} d z+c_{f} \frac{u \sqrt{u^{2}+v^{2}}}{h}=\frac{1}{h}\left(\frac{\partial h \tau_{x x}}{\partial x}+\frac{\partial h \tau_{x y}}{\partial y}\right) \\
& \frac{\partial v}{\partial t}+u \frac{\partial v}{\partial x}+v \frac{\partial v}{\partial y}+g \frac{\partial \zeta}{\partial y}+\frac{1}{h} \int_{-d}^{\zeta} \frac{\partial q}{\partial y} d z+c_{f} \frac{v \sqrt{u^{2}+v^{2}}}{h}=\frac{1}{h}\left(\frac{\partial h \tau_{y x}}{\partial x}+\frac{\partial h \tau_{y y}}{\partial y}\right)
\end{aligned}
$$

avec : $\mathrm{t}$ le temps, $\zeta(\mathrm{x}, \mathrm{y}, \mathrm{t})$ l'élévation de la surface libre, $\mathrm{h}=\zeta+\mathrm{d}$ la profondeur d'eau totale, $\mathrm{u}(\mathrm{x}, \mathrm{y}, \mathrm{t})$ et $\mathrm{v}(\mathrm{x}, \mathrm{y}, \mathrm{t})$ les vitesses et $\mathrm{q}(\mathrm{x}, \mathrm{y}, \mathrm{z}, \mathrm{t})$ la pression non-hydrostatique. 


\section{XIV èmes Journées Nationales Génie Côtier - Génie Civil \\ Toulon, 29 juin au $1^{\text {er }}$ juillet 2016}

\section{Simulations numériques}

\subsection{Localisation des sites}

Les sites d'Esquibien et de Saint-Guénolé, localisés en baie d'Audierne à la pointe de la Bretagne, présentent des potentiels prometteurs avec des puissances de houle annuelle respectives de 6,9 et $21,1 \mathrm{~kW} / \mathrm{m}$. Les longueurs utiles respectives des digues pouvant être équipées sont de 340 et 250 m, par des fonds de 2 m CM (SERGENT et al., 2014).

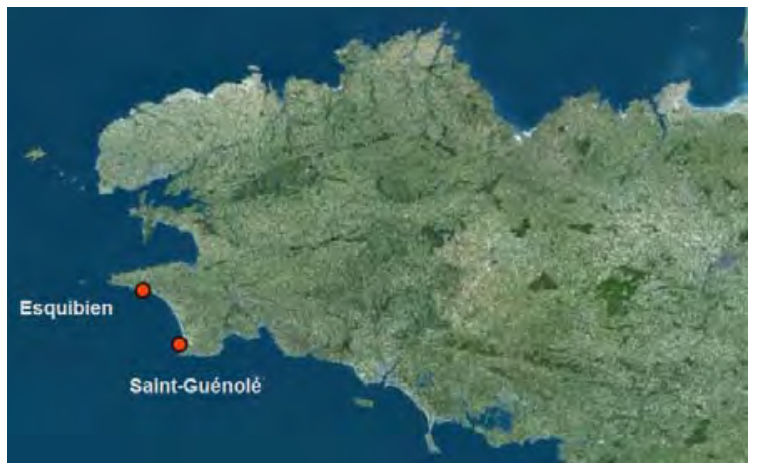

Figure 1. Localisation des deux sites, Bretagne, France (http://cms.geobretagne.fr/).

\subsection{Paramétrage des modèles numériques}

Les bathymétries obtenues à partir des cartes marines n 7147 et $n^{\circ} 6645$ du Shom sont numérisées sur les emprises des domaines d'Esquibien et de Saint-Guénolé pour une résolution de pas de 1 mètre par interpolation avec le logiciel Blue Kenue (CNRC).
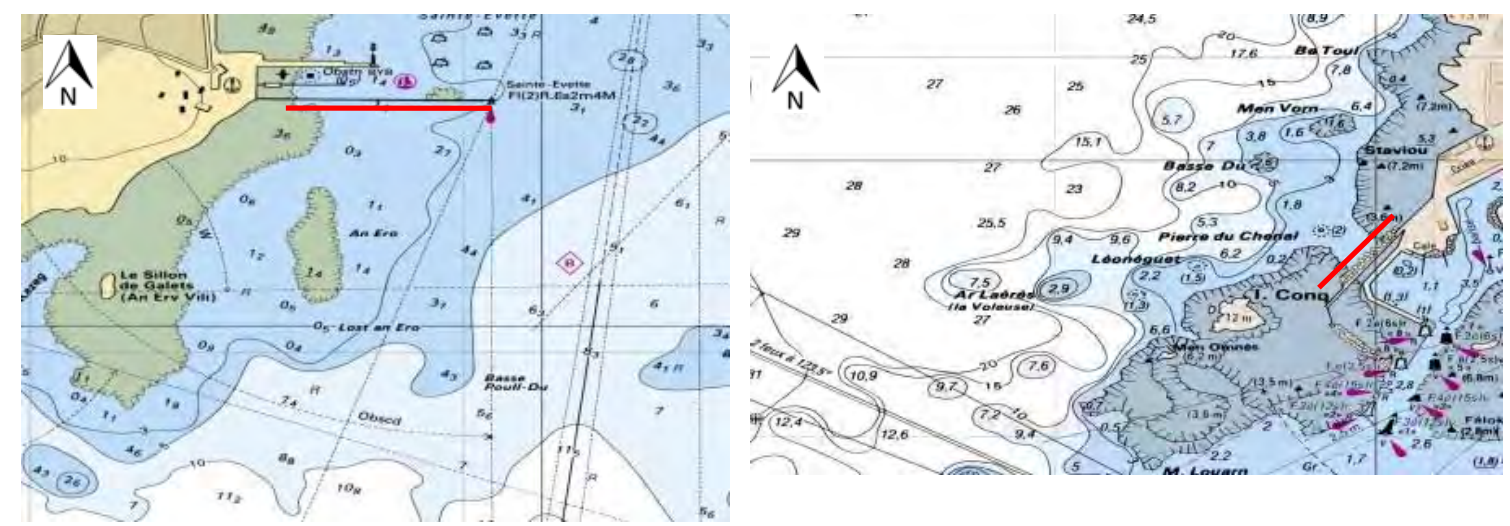

Figure 2. Cartes marines des sites d'Esquibien et de Saint-Guénolé (www.data.shom.fr).

Les paramètres numériques et physiques des modèles sont définis pour des simulations avec une couche verticale, ce qui est suffisant pour les processus de transformation des vagues en proche côtier, avec un schéma numérique explicite Keller-box (SUZUKI et al., 2011). Un coefficient de rugosité sur le fond de Manning de 0,019 est utilisé par défaut. Le pas de temps de calcul est ajusté automatiquement, dépendant des conditions 
CFL, avec un pas initial de 0,015 s pour une durée de simulation de 40 minutes.

\subsection{Création du modèle numérique du site d'Esquibien}

L'emprise du domaine de calcul du modèle est de 1,6 km selon la direction horizontale $\mathrm{x}$ et 2,1 $\mathrm{km}$ selon la direction verticale y. La grille du domaine a un pas de résolution de $4 \mathrm{~m}$ selon $\mathrm{x}$ et $2 \mathrm{~m}$ selon y. En conditions limites, des parois absorbantes sont spécifiées sur la frontière nord, à l'ouest et l'est pour absorber l'énergie des vagues. Les conditions de houle sont simulées sur la frontière entrante sud, suivant un spectre de JONSWAP et un étalement directionnel de $24,9^{\circ}$ pour différentes combinaisons de hauteurs de houle significatives (Hm0), de périodes de pic (Tp), de direction de vague et de niveaux d'eau. a) Estimation du climat de houle du site d'Esquibien

Les houles proviennent principalement du secteur $220^{\circ}$, car les houles sont réfractées par les fonds près du cap Lervily en modifiant leur direction de propagation. Trois niveaux de marée, représentant $80 \%$ des occurrences, ont été retenus à partir de l'analyse des données sur un cycle de Saros (18 ans). Les niveaux d'eau retenus sont 1,45 m pour la basse mer, 3,07 m pour la mi-marée et 4,75 m pour la pleine mer. Le choix des couples de houle a été réalisé à partir de l'analyse des données d'états de mer par $30 \mathrm{~m}$ de fond au point large W458N4796 de la base HOMERE, couvrant une période de 19 ans (1994-2012), distribuée par l'Ifremer (BOUDIERE et al., 2013). L'analyse statistique des 19 ans de données horaires reconstituées a permis de créer le tableau d’occurrences des couples (Hm0, Tp) pour en faciliter la sélection.

Tableau 1. Occurrences des couples (Hm0, Tp) en nombre d'heures ramenées sur 1 an

\begin{tabular}{|c|c|c|c|c|c|c|c|}
\hline & $\operatorname{Tp}(\mathrm{s})$ & 5 & 7 & 9 & 11 & 13 & 15 \\
\hline Hm0 (m) & & 7 & 9 & 11 & 13 & 15 & 17 \\
\hline 5.5 & 6.5 & 0.0 & 0.0 & 0.2 & 5.0 & 21.6 & 28.7 \\
\hline 4.5 & 5.5 & 0.0 & 0.1 & 3.9 & 41.0 & 78.0 & 73.3 \\
\hline 3.5 & 4.5 & 0.0 & 6.5 & 43.0 & 130.0 & 203.1 & 107.3 \\
\hline 2.5 & 3.5 & 2.9 & 75.1 & 139.7 & 316.8 & 351.9 & 123.8 \\
\hline 1.5 & 2.5 & 95.8 & 306.7 & 423.0 & 782.7 & 493.9 & 116.9 \\
\hline 0.5 & 1.5 & 243.3 & 862.2 & 1348.6 & 1228.4 & 360.0 & 111.1 \\
\hline 0 & 0.5 & 20.3 & 169.5 & 155.7 & 62.6 & 18.5 & 6.9 \\
\hline
\end{tabular}

L'examen a permis de choisir dix couples de houle (Hm0, Tp) surlignés en couleur rose en conditions limites entrantes. En résumé, l'examen du climat de houle conduit à considérer 30 simulations (10 couples de houle associés à 3 niveaux de marée). Avec un temps de calcul de 4 heures par simulation, les calculs ont duré 120 heures (5 jours).

b) Choix des points de sortie pour calculer les puissances et les spectres directionnels L'examen détaillé du site a permis de retenir 9 points de sortie pour la simulation des résultats. Ils ont été sélectionnés suivant des critères d'alignement devant la digue et de profondeurs. Les points de sortie 1, 2 et 3 sont positionnés sur l'isobathe $3 \mathrm{~m}$, les points 4,5 et 6 sur l'isobathe $5 \mathrm{~m}$ et les points 7, 8 et 9 sur l'isobathe $10 \mathrm{~m}$. 


\section{XIV ${ }^{\text {èmes }}$ Journées Nationales Génie Côtier - Génie Civil \\ Toulon, 29 juin au $1^{\text {er }}$ juillet 2016}

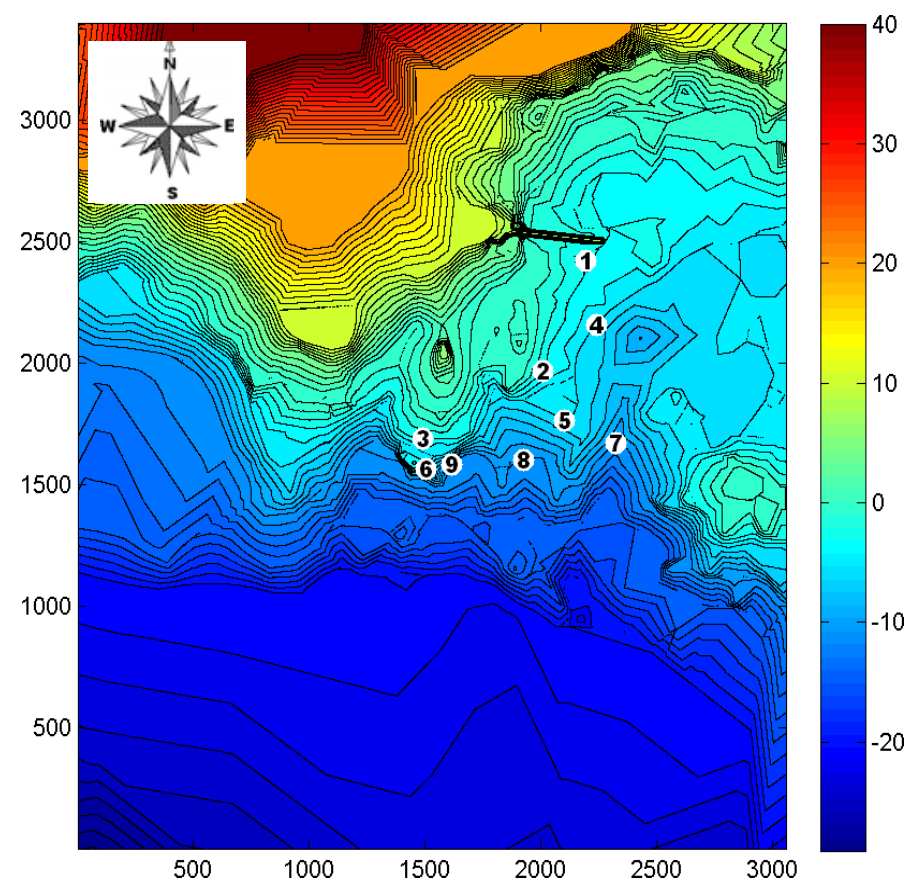

Figure 3. Carte bathymétrique d'Esquibien et emplacements des 9 points de sortie.

\subsection{Création du modèle numérique du site de Saint-Guénolé}

L'emprise du domaine de calcul est de 1,6 km selon $\mathrm{x}$ et 1,3 $\mathrm{km}$ selon y avec une résolution de $3 \mathrm{~m}$ selon $\mathrm{x}$ et $\mathrm{y}$. Les conditions de houle du secteur $270^{\circ}$ sont simulées sur la frontière entrante ouest. Le choix des couples de houle réalisé à partir par $25 \mathrm{~m}$ de fond au point large Emacop06 de la base HOMERE a été reproduit. Le calcul du climat de houle conduit à retenir 48 simulations, soit 16 couples $(\mathrm{Hm} 0, \mathrm{Tp})$ associés à 3 niveaux de marée. Avec un temps de calcul de $1 \mathrm{~h} 30$ par simulation, les calculs ont duré 72 heures. L'examen du site a permis de sélectionner 8 points de sortie, dont 5 points positionnés devant la digue et 3 autres placés plus au large sur des hauts fonds.

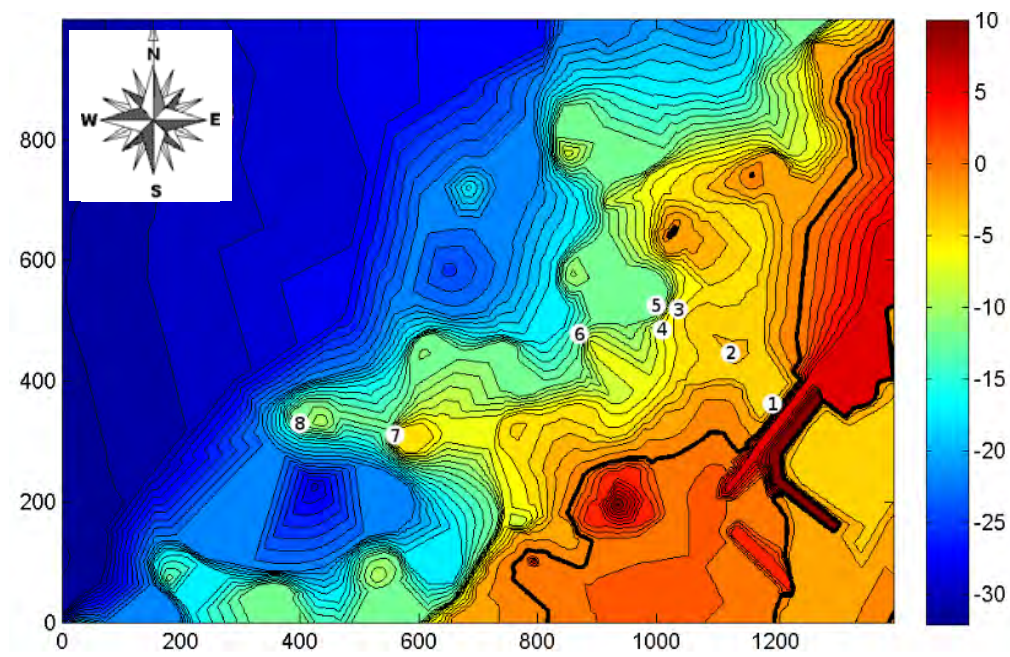

Figure 4. Carte bathymétrique de Saint-Guénolé et emplacements des 8 points de sortie. 


\section{Résultats des simulations numériques du site d'Esquibien}

\subsection{Résultats obtenus pour une simulation type du site d'Esquibien}

Les résultats obtenus par SWASH ont été traités avec MATLAB et les boîtes à outils WAFO (Wave Analysis for Fatigue and Oceanography ; www.maths.lth.se/matstat/wafo) et DIWASP (Direction-al WAve SPectra toolbox ; www.metocean.co.nz). Les résultats exploités en sortie sont l'élévation de la surface libre, la hauteur de houle, les spectres directionnels et la puissance de houle $\mathrm{P}$ calculée à partir de la formule de densité de puissance de vagues exprimée en $\mathrm{kW} / \mathrm{m}$. L'examen de l'équation suivante montre que la quantité $\mathrm{S}(\mathrm{f}) / \mathrm{f}$ est multipliée par le terme de dispersion dépendant de la fréquence et de la profondeur, ceci pour la direction moyenne de pic de vagues (EPRI, 2011) :

$$
P=\frac{\rho g^{2}}{4 \pi} \int_{0}^{\infty} \frac{S(f)}{f}\left[\left(1+\frac{2 k f d}{\sinh \left(2 k_{f} d\right)}\right) \tanh \left(k_{f} d\right)\right] d f
$$

L'exemple présenté utilise les conditions limites entrantes : hauteur de houle $\mathrm{Hm} 0$ de 2 $\mathrm{m}$, période de pic Tp de $12 \mathrm{~s}$, direction de houle de $220^{\circ}$ et niveau de pleine mer de 4,75 m. Les résultats de simulations se présentent sous la forme de tableaux, qui sont traités par un script permettant de générer les images de sortie graphique correspondantes.
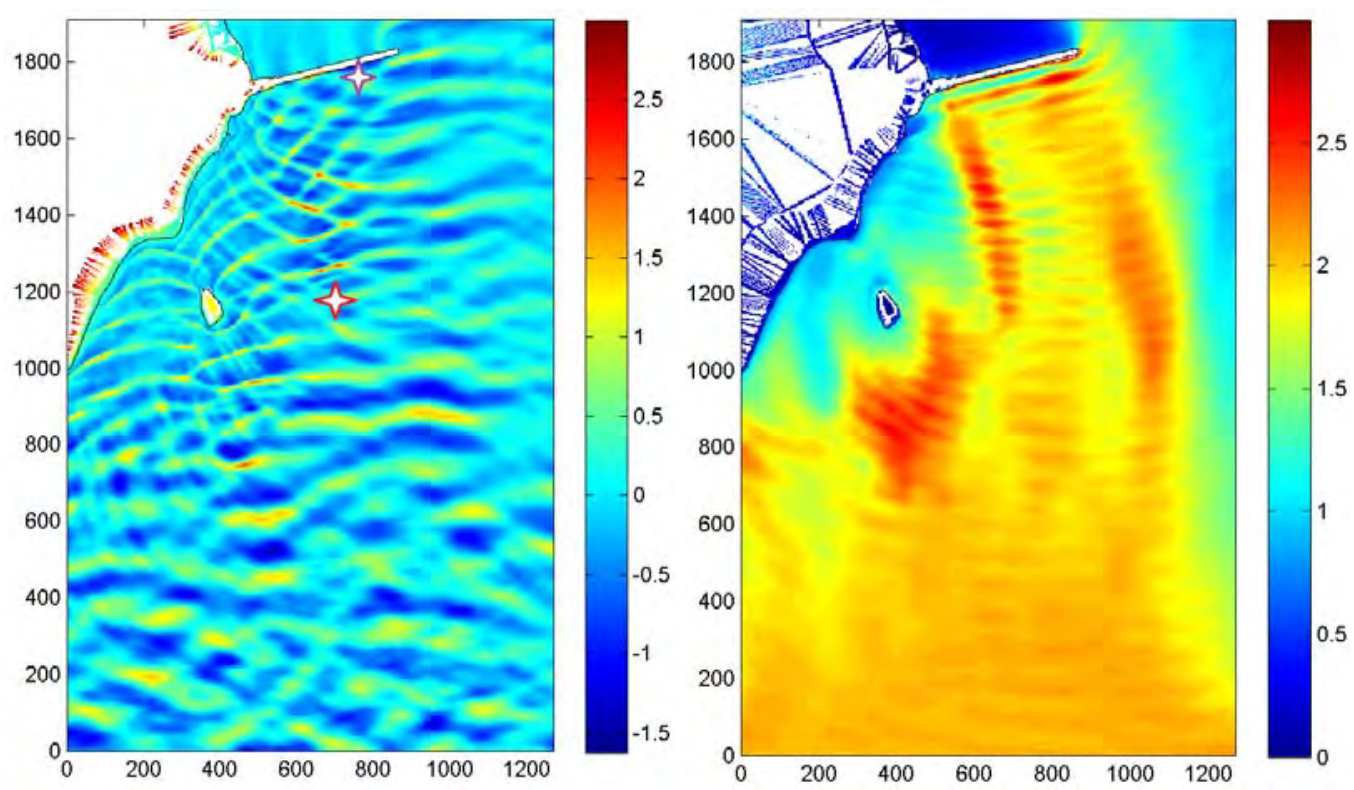

Figure 5. Résultats de l'élévation de la surface libre (à gauche) et de la hauteur de houle (à droite) pour la simulation (Hm0 : $2 \mathrm{~m}$, Tp : $12 \mathrm{~s}$, dir. : $220^{\circ}$, pleine mer 4,75 m).

La simulation a fourni des mesures d'élévation de la surface libre aux 9 points de sortie, traitées avec les scripts MATLAB pour générer les spectres directionnels (figure 6), ainsi que pour calculer les puissances de houle P correspondantes (tableau 2). 


\section{XIV vèmes Journées Nationales Génie Côtier - Génie Civil \\ Toulon, 29 juin au $1^{\text {er }}$ juillet 2016}

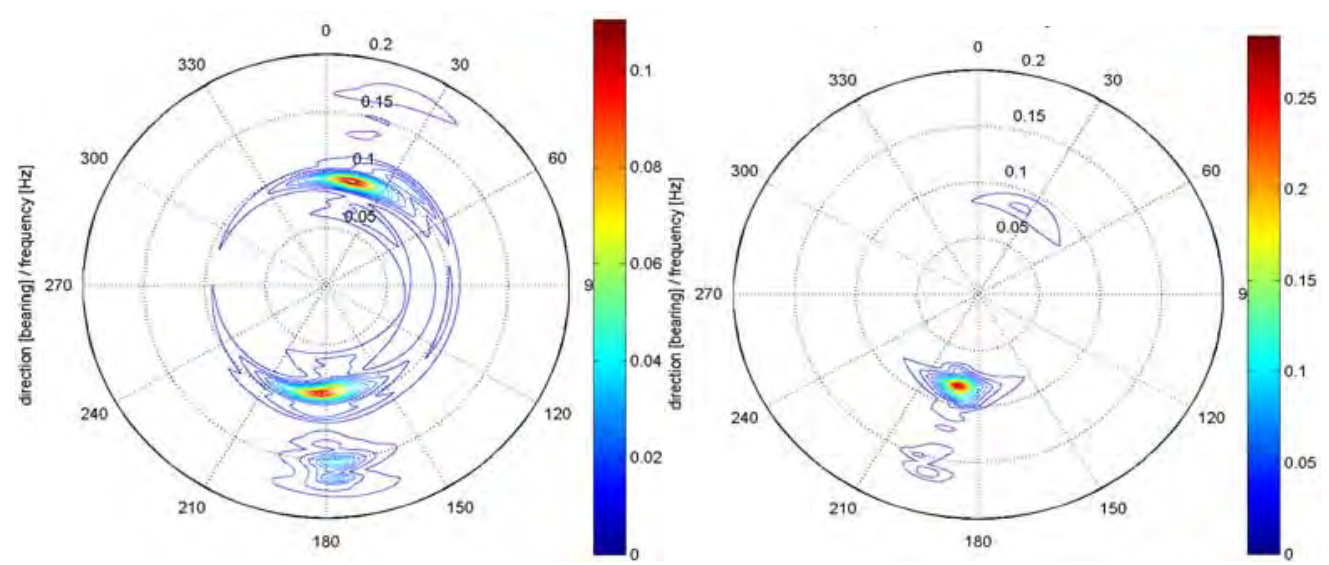

Figure 6. Résultats de spectres directionnels au point 1 (à gauche; croix violette de la figure 4) et au point 2 (à droite; croix rouge de la figure 4) pour la simulation type.

Tableau 2. Puissances de houle P calculées en chacun des 9 points de sortie-Esquibien.

\begin{tabular}{llllllllll}
\hline Point de sortie & 1 & 2 & 3 & 4 & 5 & 6 & 7 & 8 & 9 \\
\hline$P(k W / m)$ & 16,3 & 15,8 & 26,3 & 21,4 & 16,3 & 21,8 & 10,4 & 22,1 & 21,7 \\
\hline
\end{tabular}

L'observation des cartes montre les zones de concentration d'énergie, comme les zones de levage situées devant l'île aux vaches, correspondants aux points $n^{\circ} 3,6$ et 9 , plus à l'est au point $\mathrm{n}^{\circ} 4$ et près de la digue proche du point $\mathrm{n}^{\circ} 1$, avec des hauteurs de $2,7 \mathrm{~m}$.

a)Phénomène de réflexion au point $n^{\circ} 1$ près de la digue d'Esquibien

À l'extrémité de la digue, et à intervalle régulier correspondant à une demi-longueur d'onde, des variations importantes des hauteurs de houle se produisent, dû à la réflexion sur la digue. Le phénomène correspond aux interférences entre les ondes réfléchies et incidentes, créant des ventres et des nœuds. Le spectre directionnel obtenu au point $\mathrm{n}^{\circ} 1$ (figure 6 , gauche) montre que les houles réfléchies conservent leur période de pic (12 s) (COIGNARD et al., 2014). La puissance de houle disponible est calculée à partir des spectres d'élévations de la surface libre, compte tenu des probabilités d'occurrence des trois niveaux de marée et des différentes conditions de houle incidentes. Elle fournit ainsi l'énergie annuelle totale sous forme de pilonnement au point d'intérêt. Toutefois, il convient de noter que les dispositifs de récupération de l'énergie ne sont pas conçus pour utiliser exclusivement le pilonnement en un seul point. Ainsi, l'emplacement devant la digue d'Esquibien subit les effets de réflexion et de levage des houles, qui distribuent l'énergie entrante en plusieurs catégories, à savoir les houles incidentes et réfléchies, et l'onde fondamentale libre et les ondes harmoniques liées à la somme et la différence des fréquences. En considérant seulement le pilonnement, les ondes incidentes et réfléchies peuvent s'ajouter ou s'annuler en fonction de l'emplacement, de la direction de houle dominante et de la longueur d'onde. Une analyse de la conception 


\section{Thème 5 - Énergies et ressources marines}

des systèmes exigera donc de savoir comment l'énergie peut être divisée en fonction de ces caractéristiques.

b)Analyse du partitionnement des spectres directionnels de vagues

Pour y répondre, l'analyse réalisée sur les spectres directionnels pour trois niveaux de marée est présentée ici pour les conditions de houle de $2 \mathrm{~m}$ et le niveau de pleine mer. Les caractéristiques des partitions du cas (figure 7) sont données dans le tableau 3. Les partitions 1 à 3 peuvent être identifiées comme incidentes et les partitions 4 à 6 comme réfléchies. Le système de houles incidentes est à la période $12 \mathrm{~s}$, et les partitions 1 et 4 peuvent donc être caractérisées comme houles primaires (libres), et les partitions 2 et 5, la somme des fréquences des houles (liées) comme harmoniques, et les partitions 3 et 6 des harmoniques d'ordre supérieurs. Lors de la conception, il faut garder à l'esprit que la récupération de la puissance incidente diminuera en proportion de la puissance réfléchie disponible et que l'énergie des harmoniques des houles (liées) est difficile à récupérer.

Le tableau 4 indique que la puissance de la houle incidente (libre), la seule partie qui peut être aisément extraite, ne représente que le tiers et la moitié de la puissance totale observée. Pourtant, les spectres directionnels (2D) présentent généralement plus de puissance que les spectres (1D) au point de pilonnement (cf. les 2 premières colonnes).

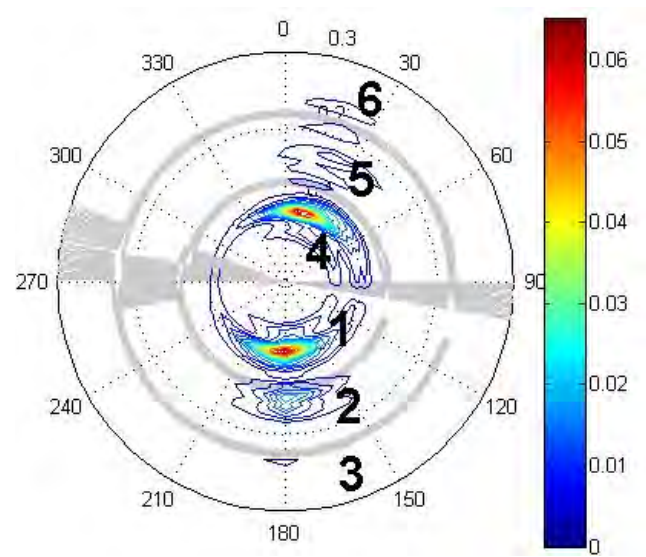

Figure 7. Résultats de partitionnement du spectre directionnel au point 1 - Esquibien.

Tableau 3. Partitionnement du spectre au point 1 (puissance totale de 22,37 kW/m).

\begin{tabular}{lllllll}
\hline $\begin{array}{l}\text { Paramètres } \\
\text { de houle }\end{array}$ & \multicolumn{2}{c}{ Système de houles incidentes } & \multicolumn{3}{c}{ Système de houles réfléchies } \\
\cline { 2 - 7 } & Syst 1 & Syst 2 & Syst 3 & Syst 4 & Syst 5 & Syst 6 \\
\hline Hm0 $(\mathrm{m})$ & 1,27 & 0,69 & 0,23 & 1,24 & 0,51 & 0,26 \\
Tp $(\mathrm{s})$ & 12,63 & 6,24 & 4,10 & 12,42 & 5,77 & 4,08 \\
$P(\mathrm{~kW} / \mathrm{m})$ & 8,83 & 2,58 & 0,29 & 8,35 & 1,39 & 0,38 \\
\hline
\end{tabular}




\section{XIV ${ }^{\text {èmes }}$ Journées Nationales Génie Côtier - Génie Civil \\ Toulon, 29 juin au $1^{\text {er }}$ juillet 2016}

Tableau 4. Résultats de puissances de houles incidente et réfléchie par niveau de marée.

\begin{tabular}{lllll}
\hline Niveau de marée & Puissance totale (1D) & Puissance totale (2D) & Incidente (libre) & Réfléchie (libre) \\
\hline Basse mer (1,45m) & 10,16 & 13,43 & 4,89 & 4,99 \\
Mi-marée (3,07m) & 16,72 & 19,58 & 9,41 & 7,15 \\
Pleine mer (4,75m) & 16,30 & 22,37 & 8,83 & 8,35 \\
\hline
\end{tabular}

4.2 Résultats de potentiel houlomoteur du site d'Esquibien

a) Les résultats des 30 simulations sont ensuite compilés dans un tableur et les données manquantes sont extrapolées à partir des résultats obtenus. Voici les résultats au point de sortie $\mathrm{n}^{\circ} 4$, situé à $100 \mathrm{~m}$ de la digue. Les cases vertes des trois tableaux 5, 6, et 7 correspondent aux résultats de puissance des simulations, et les valeurs voisines sont les valeurs extrapolées. L'énergie annuelle est ensuite calculée en multipliant les valeurs de puissance par les occurrences en nombre d'heures correspondant à chaque case du tableau d'occurrence (tableau 1), ce qui fournit trois tableaux intermédiaires d'énergie annuelle non présentés ici. Concernant le cumul des résultats par niveaux de marée, une sommation est réalisée, avec les coefficients de pondération suivants (0,3 pour la basse mer, 0,4 pour la mi-marée et 0,3 pour la pleine mer) afin de rassembler les trois tableaux d'énergie correspondants en un tableau global d'énergie annuelle (tableau 8).

Au point $n^{\circ} 4$, la somme des énergies pour les couples (Hm0, Tp) fournit une énergie E annuelle moyenne de 137,4 MWh/m, soit une puissance de houle P de 15,7 kW/m.

b)Les résultats d'énergie $E$ annuelle et de puissance de houle $P$ pour les 9 points de sortie, présentés dans le tableau 9 , montrent des variations de puissance significatives allant de 9,3 à 20,8 kW/m suivant l'emplacement sur le site.

Tableau 5. Résultats de puissance de houle $P(\mathrm{~kW} / \mathrm{m})$, niveau d'eau 1,45m (basse mer).

\begin{tabular}{rr|rrrrrr}
\hline & Tp (s) & 5 & 7 & 9 & 11 & 13 & 15 \\
Hm0 (m) & & 7 & 9 & 11 & 13 & 15 & 17 \\
\hline 5.5 & $\mathbf{6 . 5}$ & & & & & 15.27 & 8.33 \\
$\mathbf{4 . 5}$ & 5.5 & & & & 23.43 & 19.06 & 14.70 \\
3.5 & 4.5 & & & 28.00 & 24.64 & 22.86 & 21.07 \\
$\mathbf{2 . 5}$ & 3.5 & & 16.94 & 21.40 & 25.85 & 26.65 & 27.47 \\
$\mathbf{1 . 5}$ & $\mathbf{2 . 5}$ & 6.46 & 10.63 & 14.80 & 17.50 & 19.34 & 21.36 \\
$\mathbf{0 . 5}$ & $\mathbf{1 . 5}$ & 0.77 & 2.77 & 4.77 & 5.90 & 7.02 & \\
\hline
\end{tabular}

Tableau 6. Résultats de puissance de houle P (kW/m), niveau d'eau 3,07m (mi-marée).

\begin{tabular}{cc|rrrrrr}
\hline & Tp (s) & 5 & 7 & 9 & 11 & 13 & 15 \\
Hm) & & 7 & 9 & 11 & 13 & 15 & 17 \\
\hline 5.5 & $\mathbf{6 . 5}$ & & & & & 58.14 & 51.41 \\
$\mathbf{4 . 5}$ & 5.5 & & & & 56.05 & 53.92 & 51.78 \\
3.5 & 4.5 & & & 43.89 & 47.24 & 49.70 & 52.16 \\
2.5 & 3.5 & & 21.49 & 29.96 & 38.43 & 45.48 & 52.54 \\
$\mathbf{1 . 5}$ & 2.5 & 4.19 & 10.11 & 16.02 & 21.33 & 25.86 & 30.38 \\
$\mathbf{0 . 5}$ & $\mathbf{1 . 5}$ & 0.76 & 2.66 & 4.57 & 6.23 & 7.89 & \\
\hline
\end{tabular}


Tableau 7. Résultats de puissance de houle P $(\mathrm{kW} / \mathrm{m})$, niveau d'eau 4,75m (pleine mer).

\begin{tabular}{cc|rrrrrr}
\hline & Tp (s) & 5 & 7 & 9 & 11 & 13 & 15 \\
Hm0 (m) & & 7 & 9 & 11 & 13 & 15 & 17 \\
\hline 5.5 & $\mathbf{6 . 5}$ & & & & & 165.36 & 194.91 \\
4.5 & 5.5 & & & & 105.02 & 126.33 & 147.65 \\
3.5 & 4.5 & & & 46.73 & 74.25 & 87.31 & 100.38 \\
2.5 & 3.5 & & 18.80 & 31.14 & 43.47 & 48.29 & 53.12 \\
1.5 & 2.5 & 2.87 & 9.20 & 15.54 & 21.47 & 23.72 & 25.98 \\
$\mathbf{0 . 5}$ & $\mathbf{1 . 5}$ & 0.61 & 2.42 & 4.24 & 5.94 & 7.65 & \\
\hline
\end{tabular}

Tableau 8. Résultats d'énergie annuelle E (MWh/m) au point $n^{\circ} 4$ (100 m de la digue).

\begin{tabular}{cc|rrrrrr} 
& Tp (s) & 5 & 7 & 9 & 11 & 13 & 15 \\
Hm0 (m) & & 7 & 9 & 11 & 13 & 15 & 17 \\
\hline 5.5 & $\mathbf{6 . 5}$ & 0.00 & 0.00 & 0.00 & 0.00 & 1.67 & 2.34 \\
4.5 & 5.5 & 0.00 & 0.00 & 0.00 & 2.50 & 5.08 & 5.09 \\
3.5 & 4.5 & 0.00 & 0.00 & 1.72 & 6.31 & 10.75 & 6.15 \\
2.5 & 3.5 & 0.00 & 1.45 & 3.88 & 11.46 & 14.31 & 5.59 \\
$\mathbf{1 . 5}$ & 2.5 & 0.43 & 3.07 & 6.56 & 15.83 & 11.49 & 3.08 \\
$\mathbf{0 . 5}$ & $\mathbf{1 . 5}$ & 0.17 & 2.26 & 6.11 & 7.42 & 2.72 & 0.00 \\
\hline
\end{tabular}

Tableau 9. Résultats d'énergie E et de puissance $P$ des 9 points du site d'Esquibien.

\begin{tabular}{llllllllll}
\hline Point de sortie & 1 & 2 & 3 & 4 & 5 & 6 & 7 & 8 & 9 \\
\hline$E(M W h / m)$ & 99,9 & 81,9 & 135,9 & 137,4 & 123,4 & 174,4 & 83,8 & 176,2 & 181,9 \\
\hline$P(k W / m)$ & 11,4 & 9,3 & 15,5 & 15,7 & 14,10 & 19,9 & 9,6 & 20,1 & 20,8 \\
\hline
\end{tabular}

\section{Résultats des simulations numériques du site de Saint-Guénolé}

5.1 Résultats obtenus pour une simulation type du site de Saint-Guénolé

La simulation type utilise les conditions de vagues suivantes : hauteur de houle $\mathrm{Hm} 0 \mathrm{de}$ $2 \mathrm{~m}$, période de pic de $12 \mathrm{~s}$, direction de houle de $270^{\circ}$ et niveau de mi-marée de 3,07m.

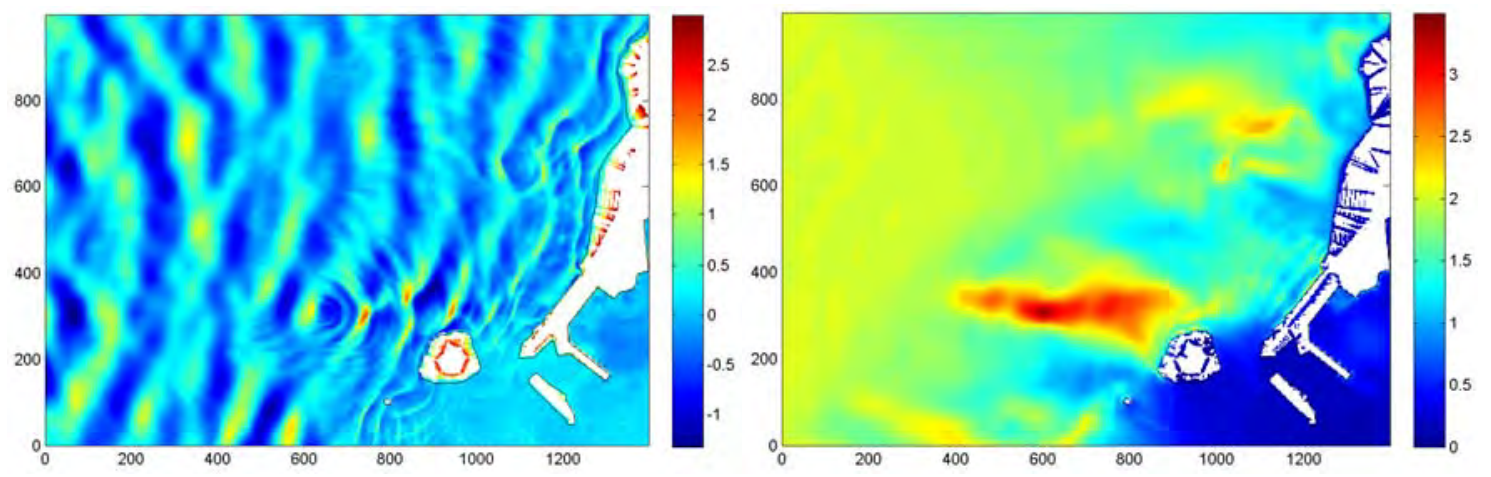

Figure 8. Résultats de l'élévation de la surface libre (à gauche) et de la hauteur de houle (à droite) pour la simulation (Hm0 : 2 m, Tp : 12s, dir. : 270, mi-marée 3,07m). 


\section{XIV ${ }^{\text {èmes }}$ Journées Nationales Génie Côtier - Génie Civil \\ Toulon, 29 juin au $1^{\text {er }}$ juillet 2016}

L'observation des deux cartes montre que les vagues se concentrent devant la zone de l'éperon rocheux, située au sud-ouest, qui s'explique par la bathymétrie du site, avec une forte remontée des fonds dans ce secteur. Les houles de plus de $3 \mathrm{~m}$ sur une distance de $400 \mathrm{~m}$, représentent un grand intérêt pour l'implantation de systèmes de récupération d'énergie. Par contre, l'énergie des vagues se dissipe davantage devant la digue, avec des hauteurs de houle de $1,5 \mathrm{~m}$ et des puissances de 3,1 à $10,2 \mathrm{~kW} / \mathrm{m}$ (tableau 10). À basse mer, le haut fond rocheux protège la digue, en concentrant et dissipant l'énergie par déferlement, comme le montre les résultats de la figure suivante.

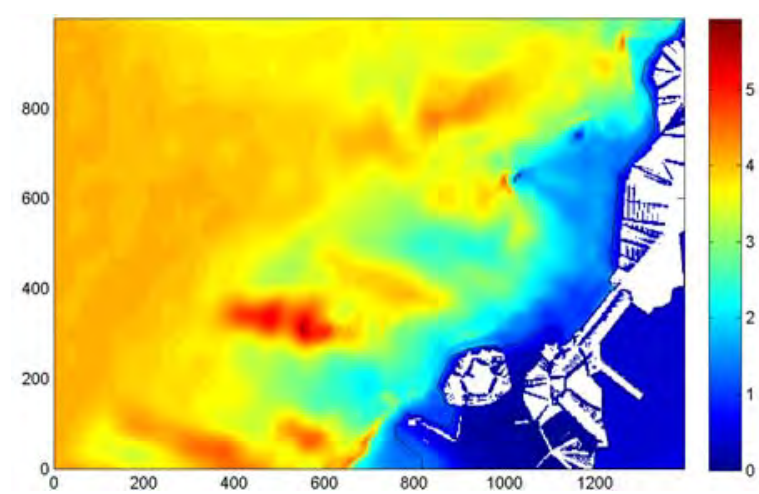

Figure 9. Résultats de hauteurs de houle pour la simulation de Saint-Guénolé à basse mer ; conditions limites entrantes (Hm0 : $4 \mathrm{~m}, \mathrm{Tp}: 14 \mathrm{~s}$, dir. : 270 ${ }^{\circ}$, basse mer 1,45 m).

Tableau 10. Puissances de houle P calculées aux 8 points de sortie - Saint-Guénolé.

\begin{tabular}{lllllllll}
\hline Point de sortie & 1 & 2 & 3 & 4 & 5 & 6 & 7 & 8 \\
\hline$P(\mathrm{~kW} / \mathrm{m})$ & 3,1 & 7,7 & 8,6 & 8,8 & 10,2 & 8,2 & 40,5 & 26,5 \\
\hline
\end{tabular}

\subsection{Résultats de potentiel houlomoteur}

Le traitement des résultats est réalisé de la même façon que pour le site d'Esquibien. Les résultats d'énergie $\mathrm{E}$ annuelle et de puissance $\mathrm{P}$ pour les 8 points de sortie présentés indiquent des variations de puissance de $2,4 \mathrm{~kW} / \mathrm{m}$ au point $\mathrm{n}^{\circ} 1$ situé devant la digue jusqu'à 33,6 kW/m au point $\mathrm{n}^{\circ} 7$ situé au niveau de la brusque remontée des fonds devant l'éperon rocheux (tableau 11).

On observe que les valeurs de puissance des points $n^{\circ} 2$ à 6 sont assez proches malgré la différence de profondeur entre ces 5 points allant de 2 à $10 \mathrm{~m} \mathrm{CM}$. Les points les plus énergétiques sont les points $\mathrm{n}^{\circ} 7$ et 8 situés au sud-ouest de la digue sur les hauts fonds, qui génèrent jusqu’à cinq fois plus de puissance houlomotrice.

Tableau 11. Résultats d'énergie et de puissance des 8 points du site de Saint-Guénolé

\begin{tabular}{lllllllll}
\hline Point de sortie & 1 & 2 & 3 & 4 & 5 & 6 & 7 & 8 \\
\hline$E(M W h / m)$ & 20,9 & 52,2 & 76,9 & 73,0 & 82,8 & 77,2 & 294,1 & 209,8 \\
\hline$P(\mathrm{~kW} / \mathrm{m})$ & 2,4 & 6,0 & 8,4 & 8,4 & 9,4 & 8,8 & 33,6 & 23,9 \\
\hline
\end{tabular}




\section{Conclusion}

Les simulations numériques de vagues avec SWASH ont permis de caractériser les conditions de vagues et le potentiel houlomoteur de deux sites de la pointe Bretagne. Les résultats de puissances de houle respectifs obtenus près des digues d'Esquibien et de Saint-Guénolé sont de 11,4 et 2,4 kW/m. Ils diffèrent des résultats obtenus en tranche 1 du projet : $6,9 \mathrm{~kW} / \mathrm{m}$ pour Esquibien et $21,1 \mathrm{~kW} / \mathrm{m}$ pour Saint-Guénolé. Ces écarts s'expliquent par des données de forçage de houle et des méthodes de calcul différentes. Pour Esquibien, le résultat obtenu ici, en tranche 2, vient confirmer l'hypothèse d'une sous-estimation du potentiel en tranche 1 due à la configuration complexe du site. De plus, le résultat de potentiel très faible près de la digue de Saint-Guénolé s'explique par la présence de l'éperon rocheux au sud-ouest immédiat, qui concentre l'énergie des houles. Un travail complémentaire, en tranche 3 de projet, est engagé en 2016 pour conforter les résultats obtenus, en analysant les mesures in-situ des capteurs de pression posés au fond durant la campagne hivernale de 2015, en effectuant des simulations avec un modèle spectral implicite WAVEWATCHIII à haute résolution et en caractérisant les incertitudes des données de forçage et des modèles numériques utilisés.

\section{Références bibliographiques}

BOUDIERE E., MAISONDIEU C., ARDHUIN F., et al. (2013). A suitable metocean hindcast database for the design of Marine energy converters, International Journal of Marine Energy, Vol. 3-4, pp e40-e52. http://dx.doi.org/10.1016/j.ijome.2013.11.010 COIGNARD J., MICHARD B., FILIPOT J.F., SERGENT P (2014). Projet EMACOP : modélisation numérique des vagues à l'approche de la digue d'Esquibien par le code SWASH, XIII ${ }^{\text {èmes }}$ JNGCGC, Dunkerque, pp 45-52. http://dx.doi.org/10.5150/jngcgc.2014.006 EPRI (2011). Mapping and assessment of the United States Ocean Wave Energy Resource. EPRI Technical report, Palo Alto, CA: 2011.1024637. 176 p.

MICHARD B., COSQUER E., DUFOUR G. (2014). Projet EMACOP : évaluation du potentiel houlomoteur de 22 sites français, XIII ${ }^{\text {èmes }}$ JNGCGC, Dunkerque, pp. 751-758. http://dx.doi.org/10.5150/jngcgc.2014.082

SERGENT P., CLEMENT A., BAUDRY V. et al. (2014). Récupération des énergies marines par dispositifs houlomoteurs, Revue Travaux n 908, pp 56-62.

SUZUKI T, VERWAEST T, HASSAN W., et al. (2011). The applicability of SWASH for modelling wave transformation and wave overtopping: A case study for the Flemish coast, ACOMEN 2011, November 2011, Liège, Belgium. pp. 1-11

ZIJLEMA M., STELLING G., SMIT P. (2011). SWASH: an operational public domain code for simulating wave fields and rapidly varied flows in coastal water. Coastal Engineering, Vol. 58, pp 992-1012. http://dx.doi.org/10.1016/j.coastaleng.2011.05.015 